\title{
Cytochrome P450 (CYP) epoxygenases as potential targets in the management of impaired diabetic wound healing
}

\author{
Huichen Zhao ${ }^{1,8}$, Jicui Chen ${ }^{2,3,8}$, Jiachao Chai ${ }^{4}$, Yuchao Zhang ${ }^{1}$, Cong Yu², Zhe Pan ${ }^{5}$, Peng Gao ${ }^{2}$, Chen Zong ${ }^{2}$, \\ Qingbo Guan ${ }^{6}$, Yuqin $\mathrm{Fu}^{7}$ and Yuantao Liu ${ }^{1}$
}

Epoxyeicosatrienoic acids (EETs) are the epoxidation products of arachidonic acid catalyzed by cytochrome P450 (CYP) epoxygenases, which possess multiple biological activities. In the present study, we aimed to explore the role and effects of CYP epoxygenases/EETs in wound healing in ob/ob mice. Full-thickness skin dorsal wounds were made on ob/ob mice and C57BL/6 control mice. The mRNA and protein expression of CYP epoxygenases were determined in granulation tissues of wounds. Effects of EETs on wound healing were evaluated. Inflammation and angiogenesis in wounds were also observed. Compared with C57BL/6 mice, the mRNA and protein expression of CYP2C65 and CYP2J6 in the granulation tissues in ob/ob mice were significantly reduced. 11,12-EET treatment significantly improved wound healing in ob/ob mice, whereas 14,15-EEZE, an EET antagonist, showed the opposite effect. 11,12-EET treatment decreased neutrophil and macrophage infiltration to the wound sites, resulting in reduced production of inflammatory cytokines, decreased MMP-9 expression, and increased collagen accumulation in the granulation tissues of ob/ob mice. In addition, 11,12-EET increased angiogenesis in the granulation tissues of wounds in ob/ob mice. These findings indicate that reduced expression of CYP epoxygenases may contribute to impaired diabetic wound healing, and exogenous EETs may improve diabetic wound healing by modulating inflammation and angiogenesis.

Laboratory Investigation (2017) 97, 782-791; doi:10.1038/labinvest.2017.21; published online 20 March 2017

Diabetic chronic wounds are a major complication in patients with diabetes mellitus that often lead to amputation and death. ${ }^{1}$ So far, there has been no efficient medication to promote wound healing in diabetic patients.

Wound healing is a highly coordinated and dynamic process regulated by numerous inflammatory factors, growth factors, and molecular events that modulate inflammatory response, cell migration, cell proliferation, extracellular matrix (ECM) deposition, and granulation formation. ${ }^{2}$ Normally, an appropriate inflammatory response is necessary for wound healing. The early phase of injury is characterized by the migration of neutrophils ${ }^{3}$ and macrophages ${ }^{4}$ to the wound sites, where they remove foreign debris and bacteria. Consequently, these cells release a variety of cytokines and growth factors that govern the formation of granulation and neovascularization. ${ }^{5}$ In diabetes, the inflammatory response at the wound site is altered, which is characterized by excessive recruitment of innate immune cells and retention of dysfunctional myeloids. ${ }^{6}$ These cells produce excessive levels of MMPs, especially MMP-9, which break down ECM components and prevents the migration and attachment of keratinocytes needed for subsequent wound healing. ${ }^{7}$ In addition, neovascularization, another key step essential for wound healing, is also impaired in diabetes. Disruption of neovessel formation leads to deficiency of oxygen, nutrients, and growth factors in the granulation tissue, prolonging the inflammatory phase $^{5}$ and reducing the proliferation of fibroblasts and the collagen synthesis. ${ }^{2}$ Many aspects of the wound-healing process are defective in diabetes, and the mechanisms underlying are still not well understood.

Department of Endocrinology, Qingdao Municipal Hospital, Qingdao, China; ${ }^{2}$ Department of Cell Biology, Shandong University School of Medicine, Jinan, China;

${ }^{3}$ Department of Blood Transfusion of Qilu Hospital, Shandong University, Jinan, China; ${ }^{4}$ Department of General Surgery, Qingdao Women and Children 's Hospital, Qingdao, China; ${ }^{5}$ Department of Endocrinology, The Second Hospital of Shandong University, Jinan, China; ${ }^{6}$ Department of Endocrinology, Provincial Hospital Affiliated to Shandong University, Jinan, China and ${ }^{7}$ Department of Nephrology, The Second Hospital of Shandong University, Jinan, China

Correspondence: Dr Liu Yuantao, PhD, Department of Endocrinology, Qingdao Municipal Hospital, 5 Donghai Street, Qingdao 266071, China or Dr Fu Yugin, MD,

Department of Nephrology, The Second Hospital of Shandong University, 247 Beiyuan Street, Jinan 250033, China.

E-mail: sduliuyuantao@163.com

${ }^{8}$ These authors contributed equally to this work.

Received 2 October 2016; revised 28 December 2016; accepted 26 January 2017 
EETs are epoxidation products of arachidonic acid catalyzed by CYP epoxygenases. EETs have four regioisomers, including 5,6-EET, 8,9-EET, 11,12-EET, and 14,15-EET. ${ }^{8}$ Accumulating evidence shows that EETs are bio-active signaling molecules that have vasodilative, anti-hypertensive, cardioprotective, renal-protective, ${ }^{9}$ anti-inflammatory, ${ }^{10}$ and proangiogenic effects. ${ }^{11}$ In vivo, EETs can be rapidly converted to less active fatty acid diols termed dihydroxyeicosatrienoic acids by soluble epoxide hydrolase (sEH). Functionally, EETs are antagonized by 14,15-EEZE. ${ }^{12}$ CYP2J and CYP2C are the major isoforms that are abundantly expressed in endothelium. ${ }^{8,9}$

A recent study showed that the plasma concentrations of stable EET metabolites were reduced in younger onset type 2 diabetes, ${ }^{13}$ suggesting that defects in EET metabolism may play a role in diabetes-associated disorders. Overexpression of

a
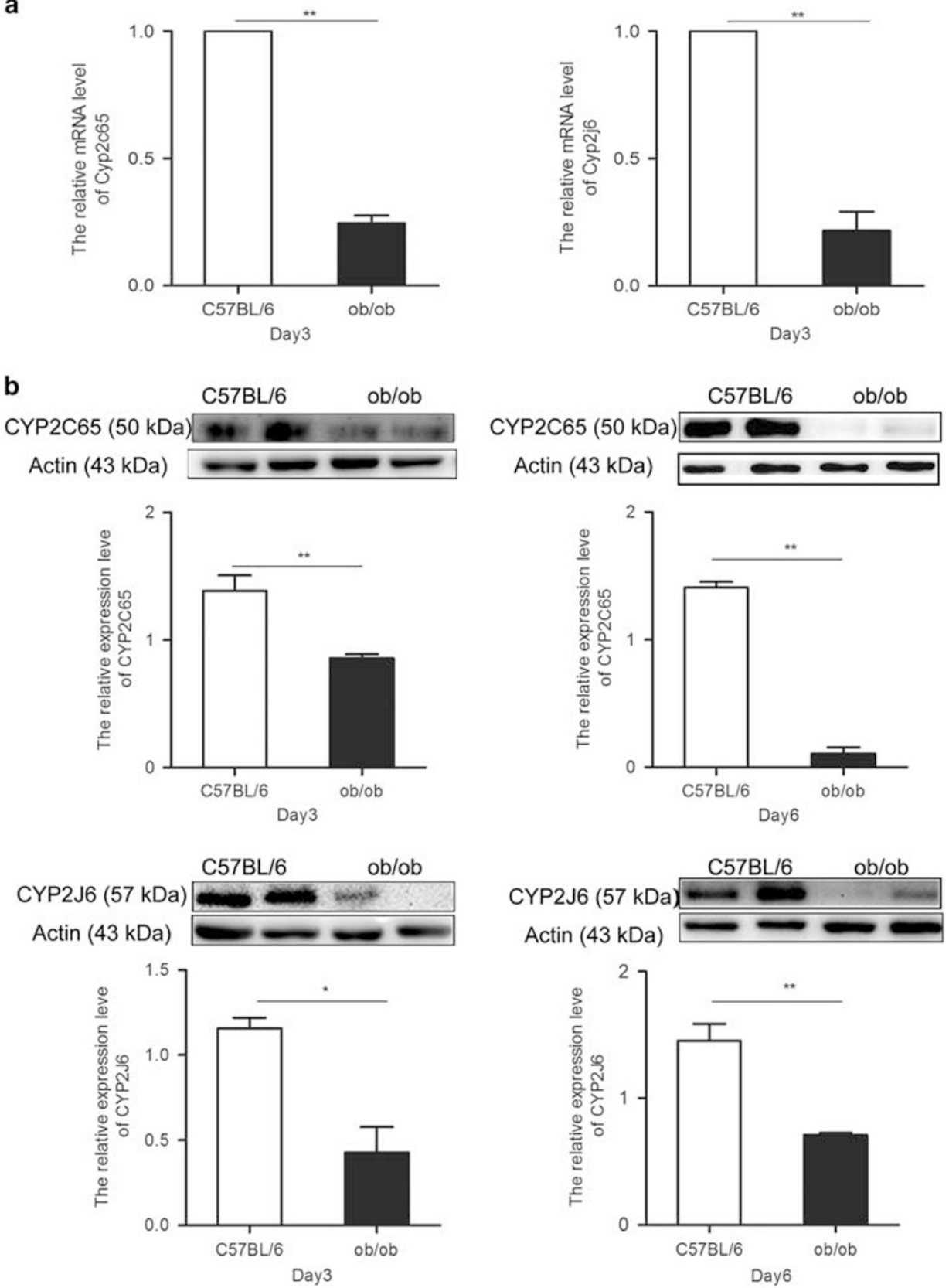

Figure 1 Reduced expression of CYP2C65 and CYP2J6 in the granulation tissues in ob/ob mice. (a) The mRNA expression levels of Cyp2c65 and Cyp2j6 in the granulation tissues in ob/ob or control mice at day 3 were evaluated by real-time PCR $(n=3$, granulation tissues of each group from three mice). Results were shown as the mean \pm s.e.m. ${ }^{* *} \mathrm{P}<0.01$, ob/ob vs C57BL/6. (b) Protein levels of CYP2C65 and CYP2J6 in the granulation tissues of wounds from ob/ob mice or C57BL/6 mice at days 3 and 6 . Representative results of western blotting, densitometric analyses of the blots were shown as bars ( $n=3$, granulation tissues of each group from three mice), and mean \pm s.e.m. ${ }^{*} P<0.05,{ }^{* *} P<0.01$, ob/ob vs C57BL/6. 
CYP epoxygenases or genetic disruption of sEH showed to attenuate metabolic dysfunction and nephropathy in diabetic mice. ${ }^{14}$ Furthermore, overexpression of Cyp2j2 prevented hepatic macrophage infiltration and restrained hepatic inflammatory signaling pathways in $\mathrm{db} / \mathrm{db}$ mice. ${ }^{15}$ It was reported that exogenous CYP-derived EETs remarkably accelerated wound epithelialization in wild type mice, possibly through the downregulation of TNF- $\alpha^{12}$ or accelerating neovascularization. ${ }^{16}$

So far, little is known about the role of CYP epoxygenases in the wound-healing process in type 2 diabetes. In the
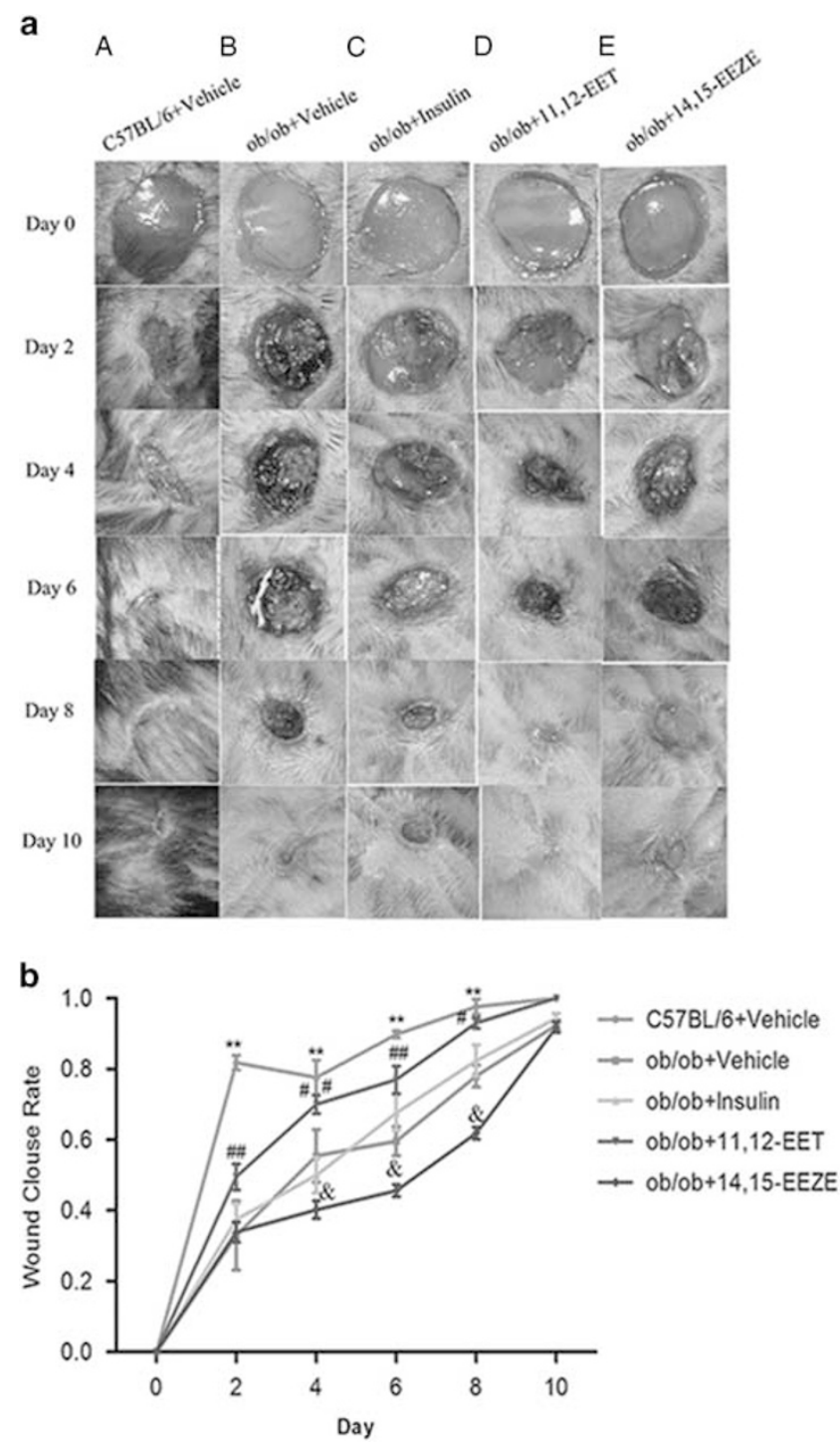

Figure 2 Effects of 11,12-EET on wound healing. Mice were randomly divided into five groups: ob/ob plus 11,12-EET, ob/ob plus 14,15-EEZE, ob/ob plus insulin, ob/ob control, and C57BL/6 control. (a) Representative photos of wound closure over time after wounding. (b) Curve graph of wound-closure rates shown as the mean \pm s.e.m. in the five groups $(n=5$, each group has five mice). ${ }^{*} P<0.01$, ob/ob vs $\mathrm{C} 57 \mathrm{BL} / 6$ control group; ${ }^{\#} P<0.05,{ }^{\# \#} P<0.01$, ob/ob vs 11,12 -EET group; ${ }^{\&} P<0.05$, ob/ob group vs 14,15-EEZE group. present study, we examined the expression of CYP epoxygenases in wounds of ob/ob mice. In addition, the effects of EETs on wound healing were evaluated, and the underlying mechanisms were explored.

\section{MATERIALS AND METHODS \\ Materials}

11,12-EET (50511) and 14,15-EE-5(Z)-E (14,15-EEZE) (10004946) were purchased from Cayman (Michigan, USA). Anti-cytochrome p450 2C11 (ab3571, 1:1500), anti-MMP-9 (ab137867, 1:1000), anti-MPO (ab9535, 1:50), anti-CD68 (ab955, 1:200), and anti-CD31 (ab28364, 1:300) antibodies were from Abcam (Cambridge, UK). Anti-CYP2J2 (sc-67276, $1: 1000)$ and normal rabbit IgG (sc-2027, 1:100) antibodies were from Santa Cruz (Cambridge, UK) product. Anti- $\beta$ actin (TA-09, 1:1000) was from Zhongshan Goldenbridge-Bio (Beijing, China). Bovine serum albumin (BSA, A0903) was from Sangon Biotech (Shanghai, China). The UltraSYBR Mixture (CW0956) and normal goat serum for blocking (CW0130) were from ComWin Biotech (Beijing, China). Primers used in this study were synthesized at the Genomics Institute of Sangon Biotech in Shanghai. Mouse TNF- $\alpha$ enzyme-linked immune-sorbent assay (ELISA) kit (E-EK282), mouse IL-6 ELISA kit (E-EK206), and mouse IL-1 $\beta$ ELISA kit (E-EK201B) were purchased from Lianke Bio (Hangzhou, China). Masson's trichrome stain kit (G1006) was obtained from Good-Biotech (Wuhan, China). Human CYP2C11 is equivalent to mouse CYP2C65, and human CYP2J2 is equivalent to mouse CYP2J6.

\section{Animal Model}

All animal procedures were conducted in accordance with guide for the care and use of laboratory animals and approved by the Animal Care and Use Committee of Shandong University. The B6.VLepob/J (leptin-deficient, ob/ob) mouse is a well-established obesity and type 2 diabetic animal models. Wild-type C57BL/6 controls and ob/ob mice have same genetic background. All mice (male, 8-12 weeks) were purchased from the Beijing HFK Bioscience Company. They have same genetic background. Mice were housed in separate cages with controlled temperature $\left(22 \pm 2{ }^{\circ} \mathrm{C}\right)$, a 12:12 light-dark cycle (lights on at $8 \mathrm{am}$ ), airflow-regulated rooms, fed with a balanced rodent diet, and water ad libitum. Blood glucose from tail veins was measured using a rapid glucose meter (LifeScan One-Touch Ultra). Mice with blood glucose level $>300 \mathrm{mg} / \mathrm{dl}$ were considered to be diabetic and used for further experiments.

Table 1 Fasting blood glucose of mice $(\mathrm{mmol} / \mathrm{l}, \boldsymbol{n}=3)$

\begin{tabular}{cccccc}
\hline & C57BL/6 & ob/ob & Insulin & $11,12-\mathrm{EET}$ & 14,15 -EEZE \\
\hline FBG & $3.67 \pm 0.39^{* *}$ & $17.43 \pm 1.9$ & $7.77 \pm 0.24^{* *}$ & $14.83 \pm 1.47$ & $17.1 \pm 1.28$
\end{tabular}

Data are presented as mean \pm s.e.m., ${ }^{* *} P<0.01$, ob/ob mice as control mice. 


\section{Full-Thickness Excisional Wounds}

Dorsal wounds creation were performed after deep anesthetized with diethyl ether. After shaving and disinfection of the dorsal skin, 9-mm-diameter full-thickness skin wounds were made with fine scissors. Gauze was used to prevent wound desiccation and mechanical irritation. Wound-closure course was analyzed by tracing the wound margin and calculating the pixel area of photographs using Photoshop CS6 software. a
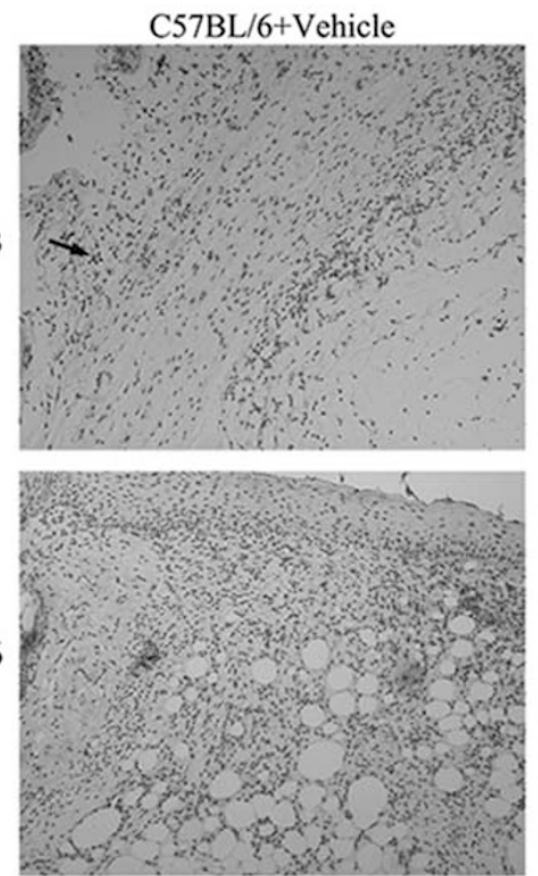

b

b
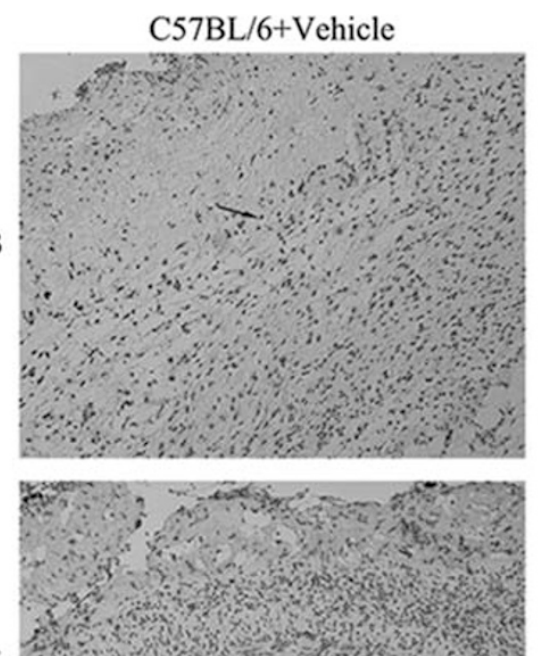

Day 6

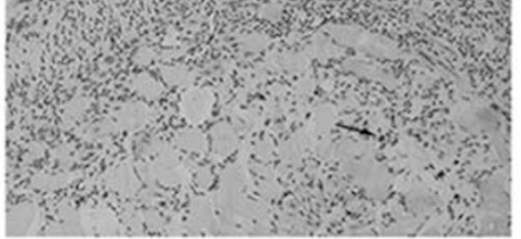

ob/ob+Vehicle
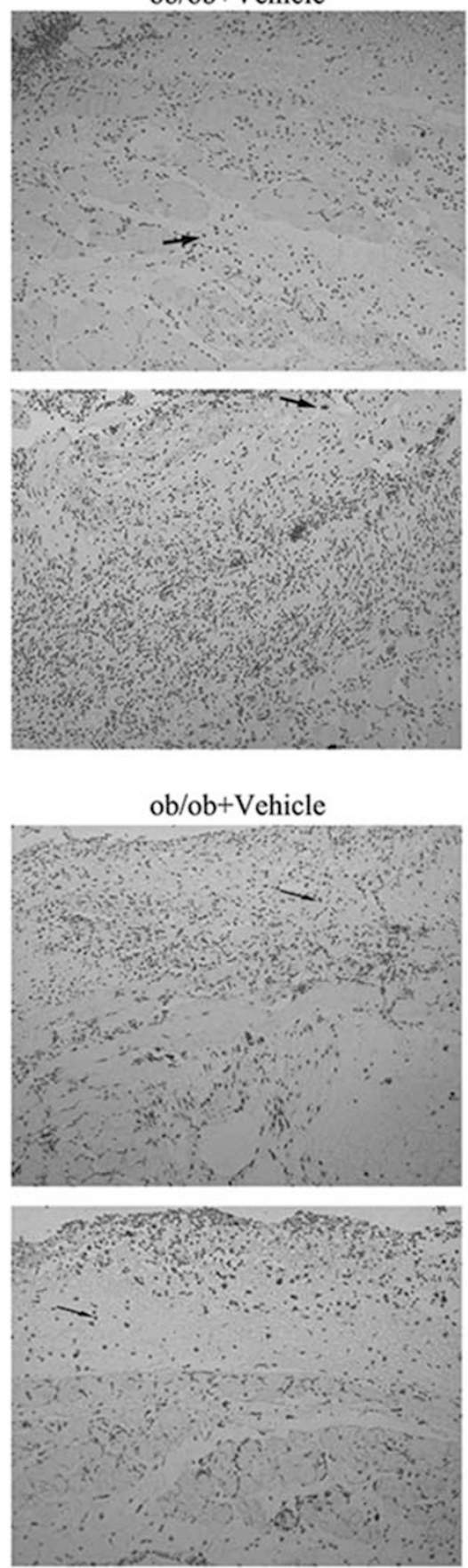
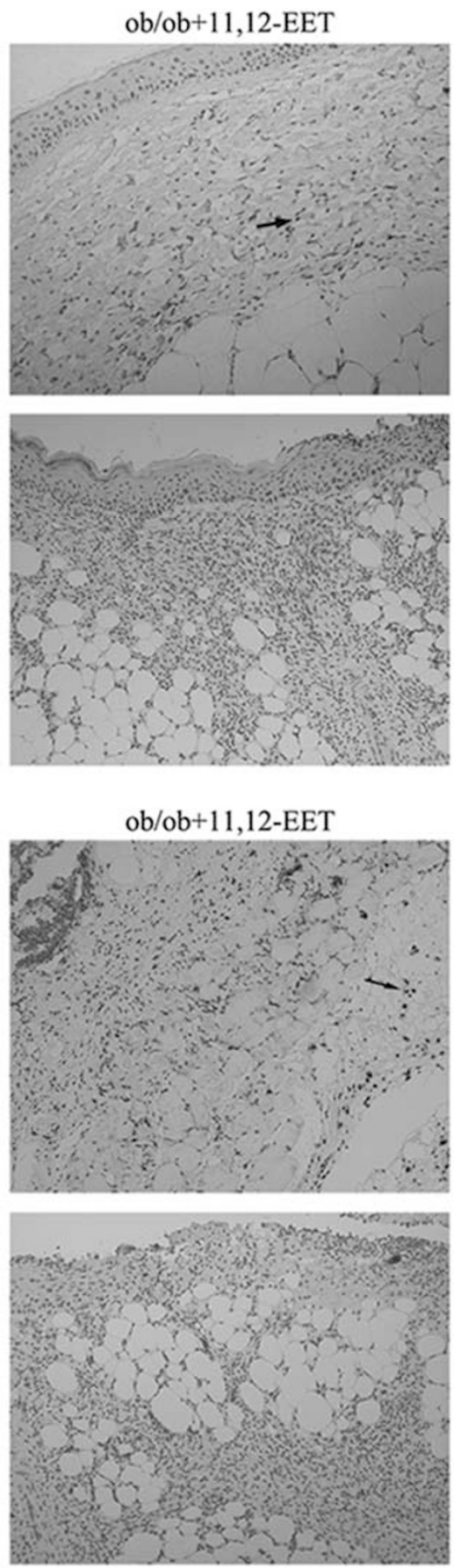

Figure 3 Effects of 11,12-EET on neutrophil and macrophage infiltration. (a) At days 3 or 6 after wounding, neutrophil infiltration evaluated by immunostining with anti-MPO antibody. Representative results of immunostaining with anti-MPO. Original magnification, $\times 100$. (b) At day 3 or 6 after wounding, immunostining with anti-CD68 antibody for macrophage. Representative results of immunostaining anti-CD68 antibodies. Original magnification, $\times 100$. (c, d) The curve charts for the average numbers of infiltrating MPO+ (c) or CD68+ (d) cells per high-power field (HPF). The results were shown as the mean \pm s.e.m. of 10 high-power fields from each mouse sample. ${ }^{* *} P<0.01$, vehicle-treated ob/ob vs control group (day 3 ); ${ }^{\# \#} P<0.01$, vehicle-treated ob/ob vs control group (day 6). 


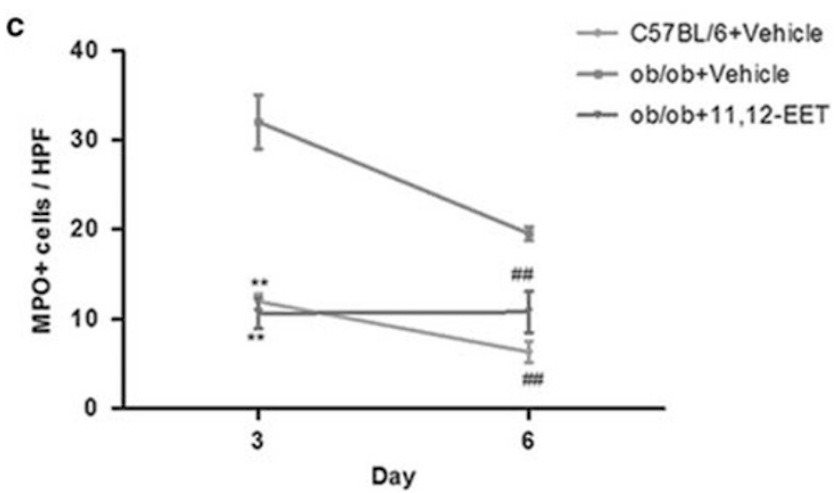

Figure 3 Continued.

Photographs of wound were captured with digital camera (Sony HX300) at $20 \mathrm{~cm}$ above the mouse dorsal every other day. Healing process was quantified by Photoshop CS6 software (Media Cybernetics), wound-closure rate was expressed as the percentage of the wound area that had healed, calculated by the following formula: $1-$ (wound area day $x /$ wound area day 0 ). (The day of wounding was specified as day 0.$)^{17}$

\section{Animal Groups and Treatment}

Mice were randomly assigned to five groups: ob/ob plus 11,12 -EET $(0.1 \mathrm{mg} / \mathrm{kg}$ per day, $n=24)$, ob/ob plus 14,15 EEZE $(0.1 \mathrm{mg} / \mathrm{kg}$ per day, $n=24),{ }^{12,18} \mathrm{ob} / \mathrm{ob}$ control (vehicle, $n=24)$, ob/ob plus insulin control $(6 \mu / \mathrm{kg}$ per day, $n=24)$, and $\mathrm{C} 57 \mathrm{BL} / 6$ mice (vehicle, $n=24$ ). These drugs were injected intra-peritoneally once daily from the day of injury until wound closure. At the end of 3 and 6 days of wounding, mice from each group were killed under diethyl ether anesthesia. Granulation tissues within the wound were collected and stored at $-80^{\circ} \mathrm{C}$ or fixed with $4 \%$ paraformaldehyde for the next experiments. Blood samples were collected from the rear caudal vein to determine the fasting blood glucose by enzymatic method at the end of day 10 .

\section{Real-Time PCR}

For RNA extraction, the $100 \mathrm{mg}$ of granulation tissues were from three ob/ob mice and three control mice at the end of 3 days of wounding and homogenized in Trizol reagent (9109, TaKaRa) with an automatic tissuelyser (Tissuelyser-24, Shanghai Jingxin Industrial Development, China) under manufacturer instructions. An amount of $2 \mu \mathrm{g}$ of total RNA was used as a template for reverse transcription with the ReverTra Ace qPCR RT kit (FSQ-101, TOYOBO). RT was accomplished at $37^{\circ} \mathrm{C}$ for $15 \mathrm{~min}$ in a final volume of $20 \mu \mathrm{l}$. The final volume contained $2 \mu \mathrm{g}$ DNaseI-treated total RNA, $4 \mu \mathrm{l} 5 \mathrm{RT}$ buffer, $1 \mu \mathrm{l}$ Enzyme Mix and $1 \mu \mathrm{l}$ Primer Mix, and nuclease-free water made up to a volume of $20 \mu$ l. Finally, enzyme was deactivated at $98^{\circ} \mathrm{C}$ for 5 min. qPCR primers were as follows: 18s rRNA-F: $5^{\prime}$-CGCGGTTCTATTTTGTT

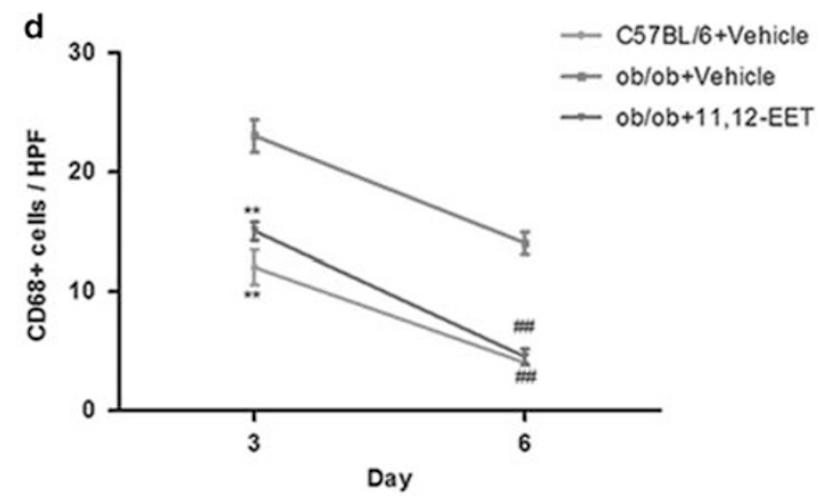

GGT-3'; 18s rRNA-R: 5'-AGTCGGCATCGTTTATGGTC-3'; Cyp2j6 rRNA-F: 5'-GGTGCCCTTGTTGTTAGCAC-3'; Cyp2j6 rRNA-R: 5'-GGCTAACAAGGAGCCGGTAG-3'; Cyp2c65 rRNA-F: 5'-AGCCCTGCACAAACTGATCT-3'; and Cyp2c65 rRNA-R: 5'-CAGAGATGTCATGCTTCCTTTGTG-3'.

Real-time quantitative PCR was implemented using UltraSYBR Mixture (with ROX) (CW0956, Cowin Biotech, Beijing, China) in CFX96TM Real-Time PCR System (Bio-Rad). The thermal cycling conditions included: $10 \mathrm{~min}$ at $95^{\circ} \mathrm{C}$ for enzyme activation; 40 cycles of denaturation for $30 \mathrm{~s}$ at $95^{\circ} \mathrm{C}$ and $1 \mathrm{~min}$ at $60^{\circ} \mathrm{C}$ for annealing and extension. Results were expressed as fold increases over control and normalized to $18 \mathrm{~s}$ RNA as previously described. ${ }^{19}$

\section{Western Blotting}

An amount of $20 \mathrm{mg}$ of granulation tissue was from three ob/ ob mice and three control mice at the end of 3 or 6 days of wounding and homogenized in $300 \mu \mathrm{l}$ RIPA buffer $(50 \mathrm{mM}$ Tris, $\mathrm{pH}$ 7.4, $150 \mathrm{mM} \mathrm{NaCl}, 1 \%$ TritonX-100, $1 \%$ sodium deoxycholate, 0.1\% SDS; Beyotime Biotechnology) and $3 \mu \mathrm{l}$ $100 \mathrm{mM}$ phenylmethylsulphonyl fluoride (PMSF). Protein concentration was quantified using BCA protein assay reagent (Sangon Biotech, China). Proteins ( $30 \mu \mathrm{g}$ ) were separated by SDS-polyacrylamide gel electrophoresis and electro-transferred onto a polyvinylidenedifluoride membrane. After blocking with $5 \%$ nonfat dry milk at room temperature for $1 \mathrm{~h}$, the membranes were consecutively incubated with primary antibodies (overnight at $4{ }^{\circ} \mathrm{C}$ ). After incubating with secondary antibodies, target proteins were detected by immunoblotting imaged with ECL chemiluminescence (Amersham Biosciences, Buckinghamshire, England). ${ }^{20}$ All measurements were conducted in triplicate. Relative densitometry was measured with NIH ImageJ 1.42 software.

\section{Immunostaining}

Granulation tissues were fixed with $4 \%$ paraformaldehyde overnight, then embedded in paraffin and sliced in $5 \mu \mathrm{m}$ with RM2016 microtome (Leica, Heidelberg, Germany). After deparaffinization, rehydration, and antigen retrieval, the 


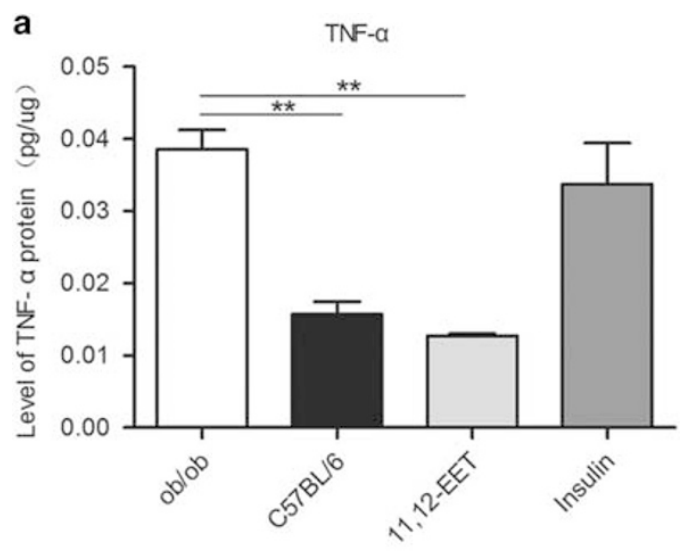

b

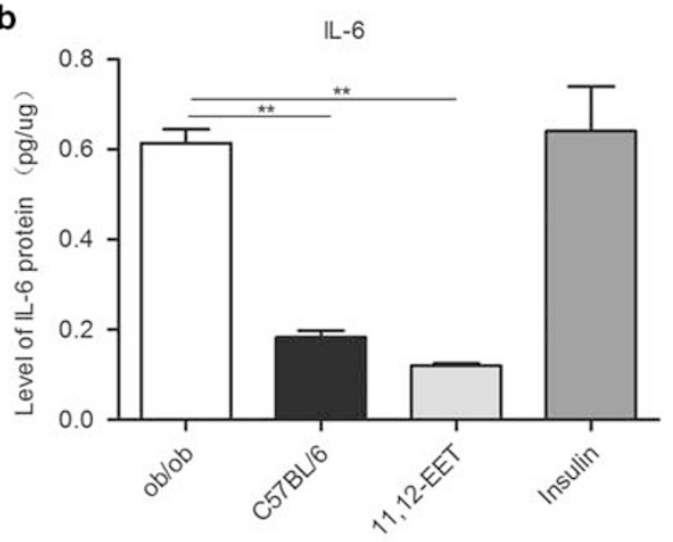

C

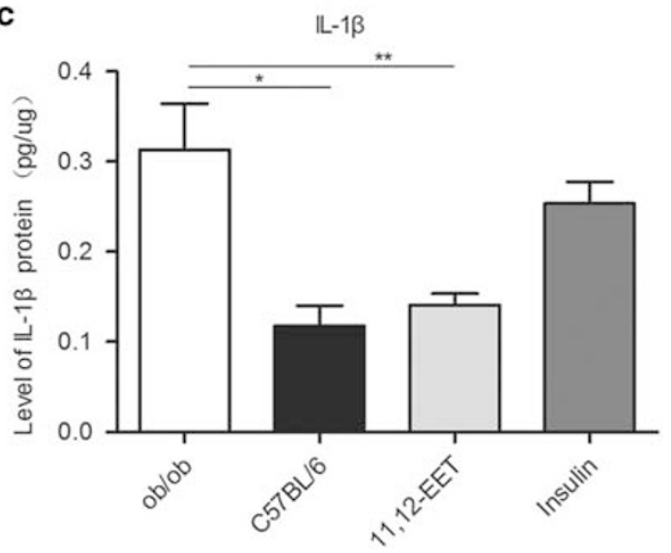

Figure 4 Effects of 11,12-EET on production of pro-inflammatory cytokines. The levels of TNF- $\alpha(\mathbf{a})$, IL-6 (b), and IL-1 $\beta$ (c) levels in granulation tissues at day 3 after wounding assessed by ELISA ( $n=3$, granulation tissues of each group from three mice), and results were expressed as the mean \pm s.e.m. ${ }^{*} P<0.05,{ }^{*} P<0.01$, vehicle-treated ob/ob as control mice.

sections were incubated with 3\% BSA for $1 \mathrm{~h}$, followed by incubation with primary antibodies (rabbit anti-CD31 1:300, rabbit anti-CD68 1:300, and anti-MPO 1:300) at $4{ }^{\circ} \mathrm{C}$ overnight. After washing with PBS, the sections were incubated with secondary antibodies. For immunofluorescence staining, sections were then stained with DAPI for $10 \mathrm{~min}$ and mounted with mounting medium, and photos were taken under a fluorescence microscope (NIKON
DS-U3) at the same instrument setting; for immunohistochemical staining, sections were incubated with horse radish peroxidase-labeled secondary antibodies, counterstained with hematoxylin, and observed under an optical microscope (Nikon, ECLIPSE CI). Normal rabbit IgG was used instead of the primary antibody as a negative control.

\section{Masson's Trichrome Staining}

After paraffin-embedded sections underwent standard deparaffinization and rehydration procedures, then stained with Masson's trichrome stain kit according to the manufacturer's instructions. Tissue sections were analyzed with an optical microscope.

\section{Enzyme-Linked Immune-Sorbent Assay}

Granulation tissues were homogenized in PBS buffer and PMSF (1:100). Debris was removed by centrifugation. Samples were analyzed immediately using mouse TNF- $\alpha$, IL-6, or IL- $1 \beta$ ELISA kits (Lianke Bio, Hangzhou, China) according to the manufacturer's instructions. Cytokine concentration was calculated against regression equation. The optimal density value of tissue samples was adjusted by protein concentration. All samples were assayed in duplicate in a single batch.

\section{Statistical Analysis}

Results were expressed as mean \pm s.e.m. Comparisons between groups were performed by one-way analysis of variance followed by the Neman-Keuls tests or two-way analysis of variance followed by the Bonferroni post tests, using GraphPad Prism 5.0 software. A value of $P<0.05$ was considered as statistically significant.

\section{RESULTS \\ CYP2C65 and CYP2J6 Expression was Decreased in ob/ob Mice}

Expression of CYP epoxygenases in the granulation tissues was determined in ob/ob mice and control mice. As shown in Figure 1a, Cyp2c65 and Cyp2j6 mRNA expression levels in the granulation tissues were significantly decreased in ob/ob mice compared with control mice $(P<0.01$, respectively). Similarly, CYP2C65 and CYP2J6 protein expression levels in ob/ob mice were significantly decreased at days 3 and 6 after wounding when compared with control mice $(P<0.05$; Figure 1).

\section{EET Accelerated Wound Healing in ob/ob Mice}

To determine whether reduced expression of CYP epoxygenases contributes to impaired diabetic wound healing, we examined the effects of 11,12-EET, a major production of CYP epoxygenases on wound healing. Results showed that wound healing in vehicle-treated ob/ob mice was slower than C57BL/6 control mice $(P<0.01$; respectively, Figure $2 \mathrm{~A}$ and B); 11,12-EET significantly accelerated wound healing in ob/ ob mice when compared with vehicle-treated ob/ob mice 
a
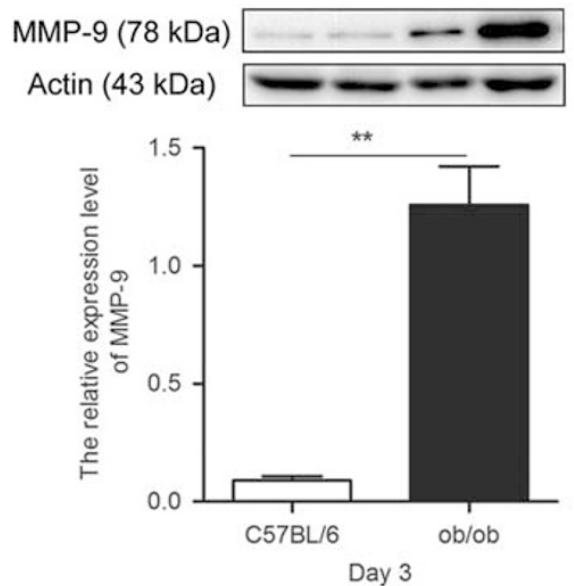

C
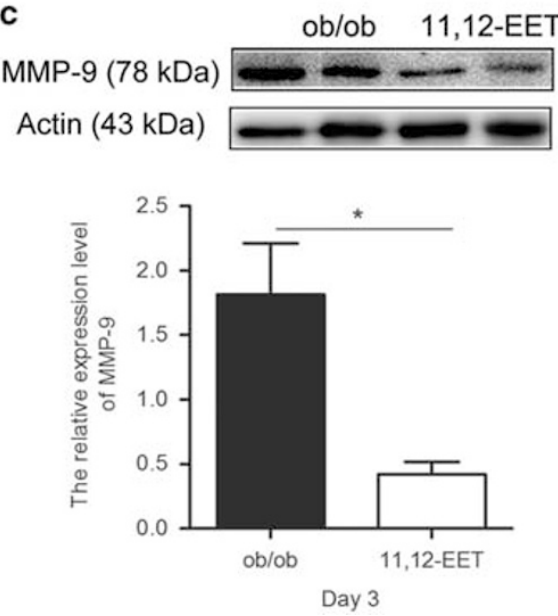

b
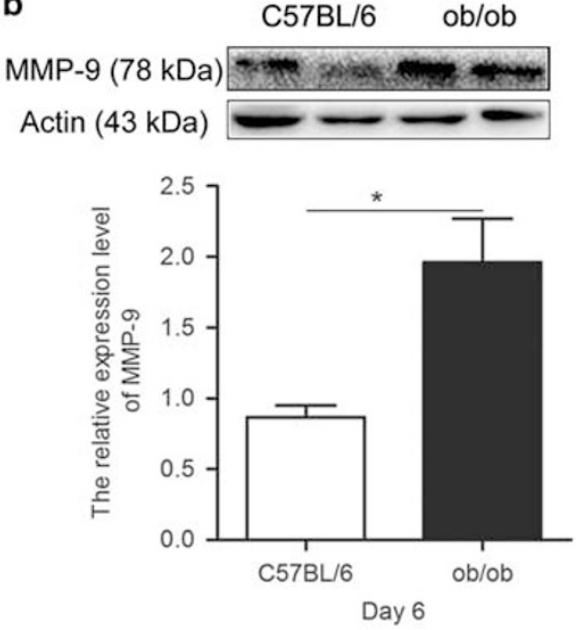

d
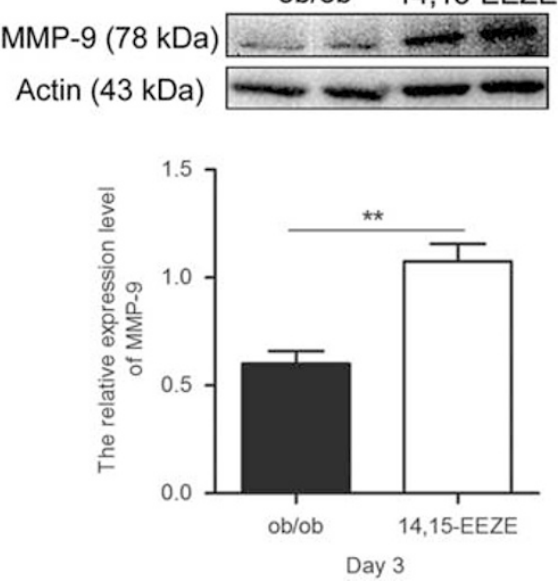

Figure 5 Effects of 11,12-EET on MMP-9 protein expression. (a, b) Protein expression of MMP-9 in the granulation tissues of wounds from ob/ob mice or C57BL/6 mice at days 3 and 6. Representative results of western blotting and densitometric analyses of the blots were shown as bar ( $n=3$, granulation tissues of each group from three mice). ${ }^{*} P<0.05,{ }^{*} P<0.01, \mathrm{C} 57 \mathrm{BL} / 6$ mice vs ob/ob mice. (c, d) Protein expression of MMP-9 in the granulation tissues of wounds from vehicle-treated ob/ob mice ( $n=3$, granulation tissues of each group from three mice), 11,12 -EET-treated ob/ob mice or 14,15 -EEZE-treated ob/ob. ${ }^{*} P<0.05$, vehicle-treated ob/ob vs 11,12 -EET-treated ob/ob; ${ }^{*} P<0.01$, vehicle-treated ob/ob vs 14,15 -EEZE-treated ob/ob.

from days 2 to $8(P<0.05$, respectively, Figure $2 \mathrm{~B}$ and $\mathrm{D})$. In contrast, 14,15-EEZE, an EET antagonist, decreased woundhealing rate in ob/ob mice compared with vehicle-treated ob/ ob mice $(P<0.05$, respectively, Figure $2 \mathrm{~A}$ and $\mathrm{B})$. Insulin significantly decreased fasting blood glucose level (Table 1) but had no obvious effect on wound healing as compared to vehicle-treated ob/ob mice $(P>0.05$, Figure $2 \mathrm{~B}$ and $\mathrm{C})$.

\section{EET Suppressed Neutrophil and Macrophage Infiltration in ob/ob Mice}

Neutrophil and macrophage infiltration was evaluated by immunostaining with anti-MPO antibody and anti-CD68 antibody, respectively. At day 3 or 6 after wounding, neutrophil and macrophage $(P<0.01$, respectively, Figure 3$)$ cell numbers in the granulation tissues of vehicle-treated ob/ ob mice were significantly higher than C57BL/6 control mice. 11,12-EET treatment significantly decreased neutrophil
$(P<0.01$; Figure $3 \mathrm{c})$ and macrophage $(P<0.01$; Figure $3 \mathrm{~d})$ infiltration when compared with vehicle-treated ob/ob mice.

\section{EET Reduced the Production of Pro-Inflammatory Cytokines in ob/ob Mice}

At day 3 after wounding, the levels of TNF- $\alpha$ (Figure 4a), IL-6 (Figure $4 \mathrm{~b}$ ) and IL-1 $\beta$ (Figure $4 \mathrm{c}$ ) in the granulation tissues of $\mathrm{ob} / \mathrm{ob}$ mice were significantly higher than $\mathrm{C} 57 \mathrm{BL} / 6$ control mice $(P<0.01$, respectively). 11,12-EET treatment significantly reduced the levels of above pro-inflammatory cytokines when compared with vehicle-treated ob/ob mice $(P<0.05)$. The levels of these pro-inflammatory cytokines were not significantly different between vehicle-treated ob/ob mice and insulin-treated ob/ob mice $(P>0.05$, respectively).

EET Decreased MMP-9 Protein Expression in ob/ob Mice Compared with C57BL/6 control mice, MMP-9 protein expression in the granulation tissues of vehicle-treated ob/ 
a

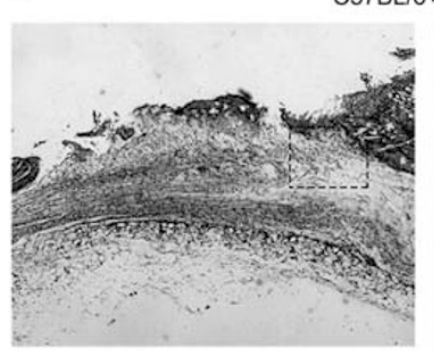

C57BL/6+Vehicle

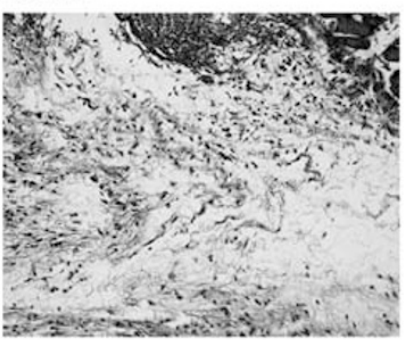

$\mathrm{ob} / \mathrm{ob}+$ Vehicle
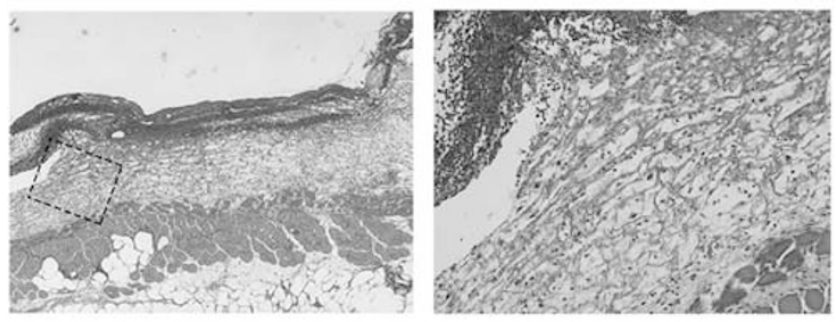

ob/ob+Insulin
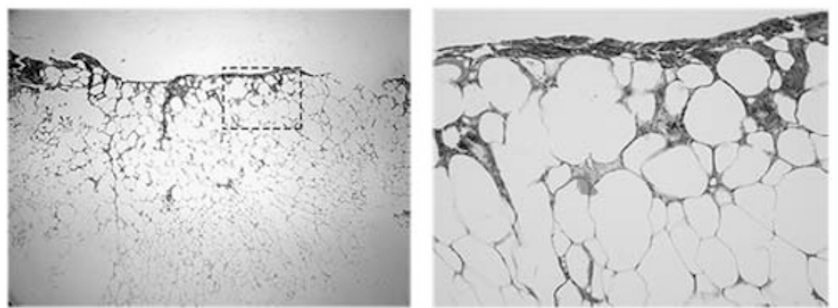
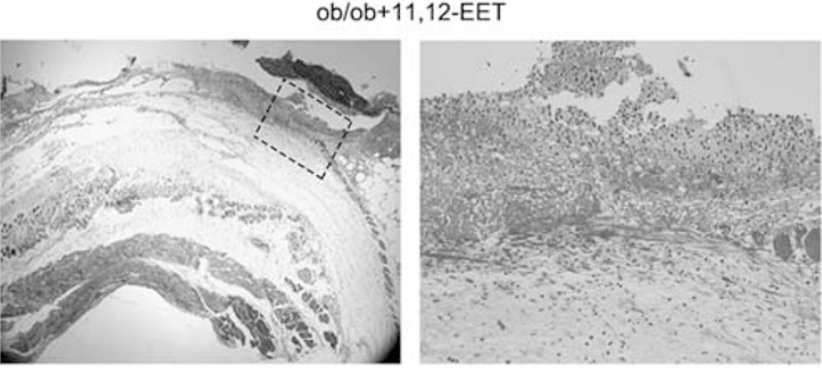

$o b / o b+14,15-E E Z E$
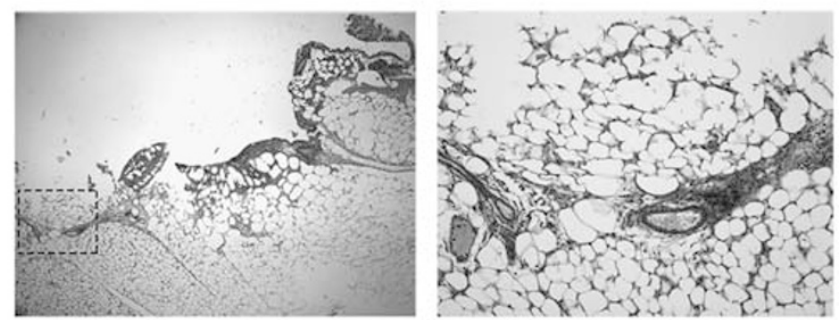

b

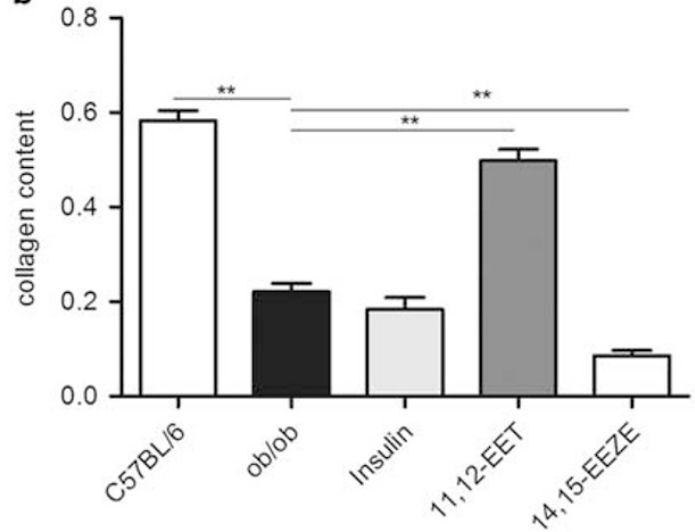

Figure 6 Effects of 11,12-EET on collagen deposition. The granulation tissues were collected at day 3 after wounding and stained Masson's trichrome. (a) Representative photomicrographs of staining with collagen content in five groups at original magnifications $\times 40$ (left) and $\times 200$ (right). The black boxes indicate the areas enlarged correspondingly in the $\times 200$ magnification panels. Collagen was stained as blue. (b) The histogram indicate relative collagen content (=total blue area/surface area). The results were shown as the mean \pm s.e.m. of 10 high-power fields from each mouse sample.

${ }^{*} P<0.01$, vehicle-treated ob/ob as control mice.

ob mice was significantly increased at days 3 and 6 after wounding $(P<0.05$; Figure $5 \mathrm{a}$ and $\mathrm{b})$. EET treatment significantly reduced MMP-9 protein expression in ob/ob mice when compared with vehicle-treated ob/ob mice $(P<0.05$; Figure 5c). In contrast, 14,15-EEZE significantly increased MMP-9 protein expression $(P<0.01$; Figure $5 d)$.

\section{EET Increased Collagen Content in Wound Skin in ob/ob Mice}

Collagen content was evaluated by Masson's trichrome staining. At day 3 after wounding, vehicle-treated ob/ob mice had reduced collagen deposition in the wound site compared with C57BL/6 control mice $(P<0.01)$. Compared with vehicle-treated ob/ob mice, EET treatment significantly increased collagen content $(P<0.01)$, whereas 14,15 -EEZE significantly reduced collagen deposition in ob/ob mice $(P<0.01)$. Insulin showed no obvious effect (Figure 6).

\section{EET Promoted Angiogenesis in the Granulation Tissues in ob/ob Mice}

At day 3 after wounding, immunostaining of fresh granulation tissues from vehicle-treated ob/ob mice showed a striking decrease in vascular-like structures (CD31-positive) compared with $\mathrm{C} 57 \mathrm{BL} / 6$ control mice $(P<0.05$; Figure 7$)$. EET treatment significantly increased the newly formed vascular-like structures in ob/ob mice when compared with vehicle-treated ob/ob mice $(P<0.01)$. Insulin alone also increased the capillary number to some extent when compared with vehicle-treated ob/ob mice, but with no statistical significance.

\section{DISCUSSION}

In the present study, we demonstrated that impaired diabetic wound healing is associated with reduced expression of CYP epoxygenases and in type 2 diabetic ob/ob mice. Exogenous 

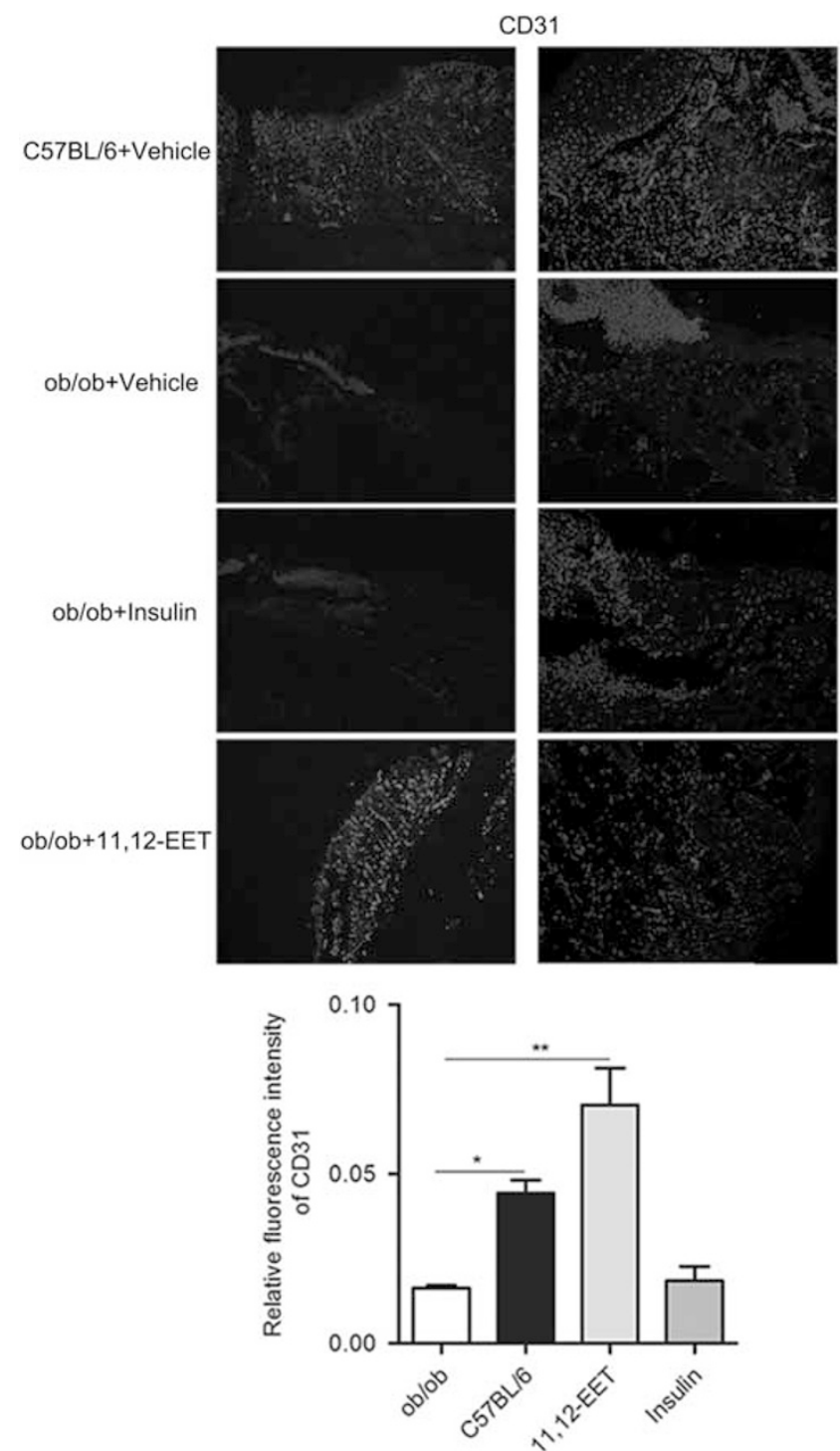

Figure 7 Effects of 11,12-EET on angiogenesis. Paraffin sections of granulation tissues from ob/ob or control mice were stained with antiCD31 antibody for capillaries. The granulation tissues were collected at day 3 after wounding. Representative photographs demonstrated vascular structure (CD31-positive) at original magnifications $\times 40$ (left) and $\times 200$ (right). Blue represents DAPI; red represents CD31. The histograms indicate relative fluorescence intensity ( $=$ total red densitometry value/ surface area). The data showed the mean \pm s.e.m. of three independent experiments, ${ }^{*} P<0.05,{ }^{*} P<0.01$, vehicle-treated ob/ob as control group.

EET significantly improved wound healing in ob/ob mice. The beneficial effect of EET was associated with its ability to attenuate local inflammation and to promote agiogenesis.

In addition to the well-recognized ability to relax smooth muscle via activation of calcium-sensitive $\mathrm{K}^{+}$channel, EETs, the products of arachidonic acid by CYP epoxygenases, have been shown to possess a variety of biological activities. ${ }^{9,10}$ Previous study showed that EETs promoted organ and tissue regeneration, ${ }^{21}$ and accelerated wound epithelialization in a mouse ear wound model. ${ }^{12,16}$ To explore the role of CYP epoxygenases/EETs in wound healing in diabetes, we first examined the expression of CYP epoxygenases in wounds of diabetic ob/ob mice. We found that the protein expression of CYP2C65 and CYP2J6 in the granulation tissues of wounds in $\mathrm{ob} / \mathrm{ob}$ mice was significantly reduced. Our results are consistent with previous findings, showing that protein expression of CYP2C11 and CYP2C23 was decreased in renal microvessels in obese Zucker rats, ${ }^{22}$ and EET metabolites were reduced in younger onset type 2 diabetes. ${ }^{13}$ These data suggested a close link between diabetes and reduced expression of CYP epoxygenases. As to how CYP epoxygenases were downregulated in ob/ob mice, the precise mechanisms remain unknown. The regulation of CYP epoxygenases seems to be complex, which involves genetic, epigenetic, and environmental factors. It was shown that CYP epoxygenase activity was not only altered in diabetes but also changed in obesity. ${ }^{23}$ It has been suggested that inflammatory cytokines may play an important role in regulation of CYP epoxygenases expression. ${ }^{24}$ Further studies are still needed to address this issue.

To determine whether reduced expression of CYP epoxygenases plays a role in impaired wound healing in diabetes, we explored the effects of 11,12-EET, a major stable product of CYP epoxygenases, and its antagonist 14,15-EEZE, on wound healing in ob/ob mice. Our data showed that 11,12-EET significantly improved wound healing in ob/ob mice, whereas 14,15-EEZE had an opposite effect. Insulin alone had little effect on wound healing despite it significantly reduced glucose levels, indicating that intrinsic defects in the wound-healing processes may exist in diabetic mice that cannot be corrected by short-term blood glucose control.

Excess inflammatory responses have been suggested to play a role in impaired wound healing. ${ }^{3,25}$ Results from immunohistochemistry experiments showed that the numbers of macrophages and neutrophils were significantly increased in the wound sites of ob/ob mice from 3 to 6 days after wounding when compared to non-diabetic mice. Accordingly, the pro-inflammatory cytokines, including TNF- $\alpha$, IL-1 $\beta$, and IL-6, were increased in the granulation tissues of diabetic mice. All of the above changes were significantly attenuated by exogenous 11,12-EET. A previous study showed that EETs may directly modulate monocyte/macrophage activity through the activation of PPAR-a. ${ }^{26}$

MMPs, derived from macrophages, are a superfamily of proteases that can degrade various kinds of ECM. As ECM provides platform for the migration and attachment of endothelial cell, fibroblasts, and keratinocytes, it plays an essential for wound healing. ${ }^{27}$ Therefore, increased production of MMP-9 due to sustained inflammation may impair wound healing by degrading ECM. ${ }^{7}$ In fact, we found that the collagen contents in the granulation tissues of wounds in ob/ ob mice were significantly reduced, which were partially prevented by 11,12-EET treatment. 
Neovascularization or angiogenesis is another key step in wound-healing process. In diabetes, angiogenesis is impaired. The proposed mechanisms include defects in endothelial cell migration, proliferation, and angiogenic factor deficiency. ${ }^{28}$ In addition, defect in endothelial progenitor cells mobilization was also suggested to play a role. In this study, we evaluated angiogenesis by immumostaining with anti-CD31 antibody. Our results showed that angiogenesis in the granulation tissues of ob/ob mice was reduced. EET treatment significantly increased the number of CD31-positive cells. Future work is needed to determine whether EET increases endothelial cell proliferation or reduces apoptosis.

Taken together, our data indicate that reduced expression of CYP epoxygenases may play a role in the pathogenesis of impaired diabetic wound healing. Exogenous EETs significantly improve wound healing in ob/ob mice possibly through attenuation of inflammatory responses and promotion of angiogenesis, providing novel therapeutic targets for diabetic ulcers.

\section{ACKNOWLEDGMENTS}

This study was supported by Nature Science Foundation of Shandong Province (no. ZR2012HL50).

\section{DISCLOSURE/CONFLICT OF INTEREST}

The authors declare no conflict of interest.

1. Icks A, Haastert B, Trautner C, et al. Incidence of lower-limb amputations in the diabetic compared to the non-diabetic population. Findings from nationwide insurance data, Germany, 2005-2007. Exp Clin Endocrinol Diabetes 2009;117:500-504.

2. Greaves NS, Ashcroft KJ, Baguneid M, et al. Current understanding of molecular and cellular mechanisms in fibroplasia and angiogenesis during acute wound healing. J Dermatol Sci 2013;72:206-217.

3. Hanses F, Park S, Rich J, et al. Reduced neutrophil apoptosis in diabetic mice during staphylococcal infection leads to prolonged Tnfa production and reduced neutrophil clearance. PLOS ONE 2011;6: e23633.

4. Miao M, Niu Y, Xie T, et al. Diabetes-impaired wound healing and altered macrophage activation: a possible pathophysiologic correlation. Wound Repair Regen 2012;20:203-213.

5. Bauer SM, Bauer RJ, Velazquez OC. Angiogenesis, vasculogenesis, and induction of healing in chronic wounds. Vasc Endovascular Surg 2005;39:293-306.

6. Wicks K, Torbica T, Umehara T, et al. Diabetes inhibits Gr-1+ myeloid cell maturation via Cebpa deregulation. Diabetes 2015;64:4184-4197.

7. Gao M, Nguyen $\Pi$, Suckow MA, et al. Acceleration of diabetic wound healing using a novel protease-anti-protease combination therapy. Proc Natl Acad Sci USA 2015;112:15226-15231.

8. Zeldin DC. Epoxygenase pathways of arachidonic acid metabolism. J Biol Chem 2001;276:36059-36062.

9. Chen C, Wang DW. CYP epoxygenase derived EETs: from cardiovascular protection to human cancer therapy. Curr Top Med Chem 2013;13:1454-1469.
10. Morisseau C, Hammock BD. Impact of soluble epoxide hydrolase and epoxyeicosanoids on human health. Ann Rev Pharmacol Toxicol 2013;53:37-58.

11. Wang $Y$, Wei $X$, Xiao $X$, et al. Arachidonic acid epoxygenase metabolites stimulate endothelial cell growth and angiogenesis via mitogen-activated protein kinase and phosphatidylinositol 3-kinase/ Akt signaling pathways. J Pharmacol Exp Ther 2005;314:522-532.

12. Sander AL, Sommer K, Neumayer T, et al. Soluble epoxide hydrolase disruption as therapeutic target for wound healing. J Surg Res 2013:182:362-367.

13. Wang $\mathrm{CP}$, Hung $\mathrm{WC}, \mathrm{Yu} \mathrm{TH}$, et al. Genetic variation in the G-50T polymorphism of the cytochrome P450 epoxygenaseCYP2J2Gene and the risk of younger onset type 2 diabetes among chinese population: potential interaction with body mass index and family history. Exp Clin Endocrinol Diabetes 2010;118:346-352.

14. Chen $G, X u R$, Wang $Y$, et al. Genetic disruption of soluble epoxide hydrolase is protective against streptozotocin-induced diabetic nephropathy. Am J Physiol Endocrinol Metab 2012;303:E563-E575.

15. Li R, Xu X, Chen C, et al. CYP2J2 attenuates metabolic dysfunction in diabetic mice by reducing hepatic inflammation via the PPAR. Am J Physiol Endocrinol Metab 2015;308:E270-E282.

16. Sander AL, Jakob H, Sommer K, et al. Cytochrome P450-derived epoxyeicosatrienoic acids accelerate wound epithelialization and neovascularization in the hairless mouse ear wound model. Langenbecks Arch Surg 2011;396:1245-1253.

17. Liu F, Chen DD, Sun $X$, et al. Hydrogen sulfide improves wound healing via restoration of endothelial progenitor cell functions and activation of angiopoietin-1 in type 2 diabetes. Diabetes 2014;63: 1763-1778.

18. Gross GJ, Gauthier KM, Moore J, et al. Effects of the selective EET antagonist, 14,15-EEZE, on cardioprotection produced by exogenous or endogenous EETs in the canine heart. Am J Physiol Heart Circ Physiol 2008;294:H2838-H2844.

19. Chen J, Ren J, Jing Q, et al. TSH/TSHR signaling suppresses fatty acid synthase (FASN) expression in adipocytes. J Cell Physiol 2015;230: 2233-2239.

20. Liu $Y$, Zhao $H$, Qiang $Y$, et al. Effects of hydrogen sulfide on high glucose-induced glomerular podocyte injury in mice. Int J Clin Exp Pathol 2015;8:6814-6820.

21. Panigrahy D, Kalish BT, Huang $S$, et al. Epoxyeicosanoids promote organ and tissue regeneration. Proc Natl Acad Sci USA 2013;110: 13528-13533.

22. Dey A, Williams RS, Pollock DM, et al. Altered kidney CYP2C and cyclooxygenase-2 levels are associated with obesity-related albuminuria. Obes Res 2004;12:1278-1289.

23. Lucas D, Farez C, Bardou LG, et al. Cytochrome P450 2E1 activity in diabetic and obese patients as assessed by chlorzoxazone hydroxylation. Fundam Clin Pharmacol 1998;12:553-558.

24. Shahabi $P$, Siest $G$, Meyer UA, et al. Human cytochrome P450 epoxygenases: variability in expression and role in inflammationrelated disorders. Pharmacol Ther 2014;144:134-161.

25. Wong SL, Demers M, Martinod K, et al. Diabetes primes neutrophils to undergo NETosis, which impairs wound healing. Nat Med 2015;21: 815-819.

26. Bystrom J, Wray JA, Sugden MC, et al. Endogenous epoxygenases are modulators of monocyte/macrophage activity. PLOS ONE 2011;6: e26591.

27. Castleberry SA, Almquist BD, Li W, et al. Self-assembled wound dressings silence MMP-9 and improve diabetic wound healing in vivo. Adv Mater 2016;28:1809-1817.

28. Feng $G$, Hao D, Chai J. Processing of CXCL12 impedes the recruitment of endothelial progenitor cells in diabetic wound healing. FEBS $J$ 2014;281:5054-5062. 